\title{
Dynamic Assessment of The Pulmonary Veins in The Cardiac Cycle of Adults Without Heart Disease by Cardiac CT
}

\section{Jing Dong}

Department of Radiology, the Affiliated Hospital of Southwest Medical University

Hui Li

Deparment of Epidemiology, Southwest Medical University

\section{Yu-Jiao Deng}

Department of Radiology, the Affiliated Hospital of Southwest Medical University

Jin-Rong zhou

Department of Radiology, the Affiliated Hospital of Southwest Medial University

\section{Jian Shu}

Department of Radiology, the Affiliated Hospital of Southwest Medical University

Jing Chen ( $\nabla$ yzqdcj@126.com )

west china hospital of sichuan university https://orcid.org/0000-0002-7200-4081

\section{Research article}

Keywords: pulmonary veins, dynamic assessment, cardiac computed tomography, cardiac cycle

Posted Date: July 20th, 2021

DOI: https://doi.org/10.21203/rs.3.rs-484866/v2

License: (c) (i) This work is licensed under a Creative Commons Attribution 4.0 International License. Read Full License 


\section{Abstract}

Purpose The purpose of this study was to explore the influence of cardiac cycle and the traditional risk factors on the four pulmonary veins (PVs) of adults and to determine the phase for measuring the maximum value of PVs.

Methods Cardiac CT was performed in 101 subjects. The diameter, area, cross-sectional angle, and coronal-section angle of four PVs in 10 phase were reconstructed and measured at $10 \%$ step from 5\%-95\% R-R interval. The differences in PVs size and spatial angles in cardiac cycles and the correlation between the indicators of four PVs and traditional risk factors were analized using two-level model.

Results All the maximum size values of the four PVs were found in $45 \%$, while the minimum values were found in $5 \%$ or $95 \%$ of cardiac phases. Gender influenced the size of three PVs-right superior pulmonary vein (RSPV), right inferior pulmonary vein (RIPV), and left inferior pulmonary vein (LSPV). The diameter of the RSPV was small in hypertensive patients and smokers. In addition, the cross-sectional angles of the left superior pulmonary vein (LIPV) changed during cardiac cycles, and age affected these changes. We found no changes in the spatial angles of the RSPV, RIPV, and LSPV, as well as the coronal-section angle of the LIPV.

Conclusions PVs ostia size of normal person varies during cardiac phases. Compared with nomal person, AF could affect the cardiac phase in which the maximum and minimum of PVs is, and it may lead to a reduction of the PVs' size slightly.

For life science journals only.

\section{Introduction}

The pulmonary veins (PVs) are large blood vessels that transport blood from the lung to the left atrium (LA), and are the only veins rich in oxygen blood flows, including right superior pulmonary vein (RSPV), right inferior pulmonary vein (RIPV), left inferior pulmonary vein (LSPV) and left superior pulmonary vein (LIPV). In recent years, the involvement of the PVs in several pathogenetic processes has increasingly been recognized.[1] The primary PVs stenosis associated with normal PVs is a rare anomaly, its severity ranges from mild discrete stenosis at the venous atrial node and PVs stenosis to the venous atresia. And involving one or more PVs can lead to severe pulmonary arterial hypertension and right ventricular failure, which could lead to death though repaired.[2] In addition, hemodynamic evaluation shows that pulmonary arterial hypertension is proportional to the increase in PVs pressure, which ultimately leads to cor pulmonale. $[3,4]$ The PVs also play a role in the onset of atrial arrhythmias, such as atrial fibrillation (AF).[5]

In recent years, several studies have explored the PVs ostia size of AF patients in radiology.[6-16] However, the focus of PVs study is on patients with AF or other cardiac disease at present, and there is 
very little study on the characteristics of normal population PVs. Only knowing the characteristics of PVs in the normal population, we can research the PVs disease better.

As we all know, PVs' size change significantly between end-systole and end-diastole,[10] but to date, no research has investigated the change of PVs size or spatial angles of normal person in detailed cardiac cycles. Hence, this study primarily aims to get more information about normal PVs--assess the dynamic changes of the size and spatial angles of normal person in 10 cardiac phases. And to the best of our knowledge, this is the first study about the dynamic changes of PVs' size and spatial angles of normal person, and it could be the basic of studying the PVs related disease better in the future.

\section{Materials And Methods}

\section{Study population}

In this study, we collected 110 imaging data of volunteers who underwent cardiac CT between June 2019 and August 2019. The inclusion criteria were as follows: (i) subjects who passed the image quality score (graded $\geq 3$ points). [Of note, the image quality scoring criteria were as follows: $1=$ the image has no diagnostic value; 2 = all or part of the cardiac cycle image has severe artifacts; $3=$ the image has a few artifacts and exerts little effect on the display of the PVs; 4 = each phase image is clear, without artifacts.] (ii) subjects' 256-slice cardiac CT scans were negative, and all had sinus heart rate (heart rate, 58-96 bpm). The exclusion criteria were as follows: subjects with AF, pulmonary hypertension, or any other cardiovascular disease that causes left atrial changes such as congenital heart disease and heart valve disease, contrast agent allergy, respiratory dysfunction, and renal or cardiac insufficiency. Finally, we enrolled 101 subjects (51 males and 50 females) in this study.

In the final data statistical analysis, based on the prevention and control of adult overweight and obesity issue, grouping by the body mass index $\left(\mathrm{BMI}, \mathrm{kg} / \mathrm{m}^{2}\right)$ was as follows: group 1 , underweight, $\mathrm{BMI}<18.5$; group 2, normal weight, BMI 18.5-23.9; group 3, overweight, BMI 24.0-27.9; and group 4, obesity, BMI $\geq 28.0$. Grouping by age was as follows: group 1 , youth group, $18-45$ years; group 2 , middle-aged group, 46-69 years; and group 3, elderly group $>69$ years.

In accordance with the 1964 Declaration of Helsinki and its later amendments, the institutional review board in our hospital reviewed our study. Because of the retrospective nature of the study, we were not required to get signed subjects informed consent.

\section{Cardiac CT}

All subjects underwent strict breathing training before the examination and were instructed to lie down during the examination; they were asked not to take $\beta$-blockers, such as metoprolol, before the examination. All subjects were scanned by a 256-slice scanner (Philips Brilliance iCT; Philips Medical Systems, Cleveland, $\mathrm{OH}$ ) with retrospective ECG; the scanning range was precisely from the bifurcation of the pulmonary artery to the level of the diaphragm with a thickness of $0.5 \mathrm{~cm}$. During scanning, a 
contrast agent iodixanol (1.0-1.2 mL/kg; General Electric Pharmaceutical Co., Ltd., Shanghai, China) was injected using contrast agent monitoring technology, and the CT threshold of ROI was set at $110 \mathrm{HU}$. Of note, acquisitions were obtained during a single breath-hold after the injection. Scan parameters were as follows: to decrease the radiation dose of scan, the scan voltage was adjusted per the patients' weight and $\mathrm{BMI}$, using automatic tube current technology. The tube voltage was $120 \mathrm{kV}$ for subjects weighing $\geq 75 \mathrm{~kg}$ and $100 \mathrm{kV}$ for subjects weighing $<75 \mathrm{~kg} ; 1200$ mas/slice for subjects with $\mathrm{BMI} \geq 24$, and 900 mas/slice for subjects with $\mathrm{BMI}<24$.

\section{Image processing and measurement methods}

The original images were reconstructed from $5-95 \%$ cardiac phases at $10 \%$ intervals. The reconstructed images were transferred to the post-processing software (IntelliSpace Portal Workspace; Philips) and loaded into the cardiac viewer application, which enabled three perpendicular axes to be adjusted simultaneously to attain perpendicular imaging planes for measurement. Two experienced radiologists (with 2- and 5-year experience in the interpretation of cardiac CT images), blinded to clinical data, independently reviewed the CT images on a dedicated post-processing workstation. They measured the diameter, area, cross-sectional angle, and coronal-section angle of the four PVs ostia. As shown in Fig. 1, we measured the diameter and area of the sinus ostium at the PVs ostia, followed by measuring the cross-sectional and coronal-section angles of the PVs ostia, respectively.

\section{Statistical analysis}

All statistical analyses in this study were performed using by MLwin software (version 2.36; University of Bristol, Bristol, The United Kingdom). We computed descriptive statistics, such as mean and standard deviation, for continuous variables. Based on the data characteristics, we built a two-level model. The dependent variables of each observation index included area, maajor axis, cross-sectional and coronalsection angle; $a=0.05$ was set as the inspection level. Cardiac phase was the first-level covariate, while gender, age, BMI, blood pressure, blood lipid, blood sugar, smoking, and drinking were second level covariates; only the statistically significant covariates were retained in the final model. Furthermore, considering interaction effects between various covariates and cardiac phases, we introduced interaction items in the final model; the interaction items that were not statistically significant after the introduction were eliminated.

\section{Results}

\section{Baseline characteristics}

In this study, we enrolled 101 subjects. Table 1 shows the clinical characteristics of the study population. Of all, 24 subjects had hypertension, 35 had hyperlipemia, and 18 had hyperglycemia. In addition, the number of smokers and drinkers was 25 and 10, respectively.

\section{Size of the four PVs}


For the RSPV, the maximum and minimum values of diameter were observed in $45 \%(19.91 \pm 3.26 \mathrm{~mm})$ and $5 \%(18.02 \pm 2.93 \mathrm{~mm})$ of cardiac phases, respectively, while the maximum and minimum values of area were observed in $45 \%(275.38 \pm 83.29 \mathrm{~mm})$ and $95 \%(196.33 \pm 64.18 \mathrm{~mm})$. The maximum and minimum values of RIPV diameter were observed in $45 \%(17.04 \pm 2.46 \mathrm{~mm})$ and $95 \%(15.30 \pm 2.48 \mathrm{~mm})$, while the maximum and minimum values of area were observed in $45 \%(210.74 \pm 58.80 \mathrm{~mm})$ and $95 \%$ $(169.92 \pm 54.11 \mathrm{~mm})$, respectively. The maximum and minimum values of LSPV diameter were observed in $45 \%(18.93 \pm 2.78 \mathrm{~mm})$ and $95 \%(16.68 \pm 2.62 \mathrm{~mm})$, while the maximum and minimum values of area were observed in $45 \%(224.22 \pm 62.53 \mathrm{~mm})$ and $5 \%(169.21 \pm 48.55 \mathrm{~mm})$, respectively. For the LIPV, the maximum and minimum values of diameter were observed in $45 \%(16.52 \pm 2.55 \mathrm{~mm})$ and $5 \%(15.11 \pm$ $2.23 \mathrm{~mm})$, while the maximum and minimum values of area were observed in $45 \%(154.84 \pm 60.52 \mathrm{~mm})$ and $5 \%(126.93 \pm 41.23 \mathrm{~mm}$ ), respectively (Tables 2 and 3$)$. The diameter and area of the four PVs ostia in cardiac phases varies significant (Fig. 2).

We used the quadratic curve model to fit the changes of the four PVs ostia diameter and area with cardiac phases; the covariates were screened one by one, and only the statistically significant factors were included in the final model to determine the correlation between traditional cardiovascular risk factors and PVs ostia (Suppl. Tables 1-12). Gender influenced the size of three PVs, like RSPV, RIPV, and LSPV. For the RSPV, RIPV, and LSPV, at the initial cardiac phase, the average level of the PVs' size between individuals of different genders was different; changes in the PVs differed at different cardiac phases, and the rate of change was also different. In addition, blood pressure and smoking affected the RSPV size, and the RSPV diameter was small in patients with hypertension and smokers. We found no effect of age, blood fat, blood sugar, BMI, and drinking on the PVs' size. Moreover, factors affecting changes in the three PVs ostia size didn't be found entirely. For the LIPV, no statistically significant variables were found.

\section{Spatial angles of PVs}

For the RSPV, the maximum and minimum values of cross-sectional angles were observed in $5 \%(18.30 \pm$ $8.29 \mathrm{~mm})$ and $45 \%(16.41 \pm 7.55 \mathrm{~mm})$ of cardiac phases, respectively, while the maximum and minimum

values of coronal-section angles were observed in $5 \%(25.10 \pm 8.73 \mathrm{~mm})$ and $35 \%(23.39 \pm 8.37 \mathrm{~mm})$. The maximum and minimum values of RIPV cross-sectional angles were observed in $45 \%$ (30.28 $\pm 11.19 \mathrm{~mm})$ and $5 \%(29.30 \pm 11.69 \mathrm{~mm})$ respectively, while the maximum and minimum values of coronal-section angles were observed in $5 \%(18.06 \pm 9.93 \mathrm{~mm})$ and $65 \%(17.34 \pm 9.38 \mathrm{~mm})$. And the maximum and minimum values of LSPV cross-sectional angles were observed in $45 \%(17.17 \pm 8.31 \mathrm{~mm})$ and $85 \%(15.75$ $\pm 8.21 \mathrm{~mm}$ ) respectively, while the maximum and minimum values of coronal-section angles were observed in $45 \%(33.09 \pm 12.30 \mathrm{~mm})$ and $75 \%(31.10 \pm 11.68 \mathrm{~mm})$. The maximum and minimum values of LIPV cross-sectional angles were observed in $45 \%(22.18 \pm 9.06 \mathrm{~mm})$ and $5 \%(20.18 \pm 8.76 \mathrm{~mm})$, while the maximum and minimum values of coronal-section angles were observed in $55 \%(20.59 \pm 10.01 \mathrm{~mm})$ and $85 \%(19.38 \pm 9.02 \mathrm{~mm})$, respectively (Tables 2 and 3$)$. 
As the changing trend of the RSPV, RIPV, and LSPV sptaial angles was a straight line, they were not included in the model fitting stage, neither did the LIPV coronal-section angle. Figure 2 shows the difference in the cross-sectional angle of the LIPV. We used a quadratic model to fit the trend of the LIPV cross-sectional angle with cardiac phases. The covariates were screened one by one; as the fixed effects of age were statistically significant, they were included in the final model. Suppl. Suppl. Tables 13 and 14 show the final model. In the initial phase, the average value of LIPV's cross-sectional angle among different-age individuals was different. In different cardiac phases, different-age individuals exhibited different changes in LIPV's cross-sectional angle, and individuals with different ages had a different speed of the LIPV's cross-sectional angle change. Of note, factors affecting changes in LIPV's crosssectional angles were not found entirely.

\section{Discussion}

\section{Main findings}

The statistical methods used in this study involved models that considered several factors, which makes it the most extensive study of normal PVs to date. This study reveals that the PVs ostia size is variable during cardiac cycles for normal person. All the maximum values of the four PVs' size were observed in $45 \%$ of cardiac phases, while the maximum values of the RSPV angles and RIPV coronal-section angles, were observed in 5\%. In addition, the maximum values of the LSPV angles, LIPV cross-sectional angles, and RIPV cross-sectional angles were observed in $45 \%$; however, the maximum values of the LIPV coronal-section angles were observed in $55 \%$.

\section{Size of PVs}

In our study, the maximum PVs size was in $45 \%$ of cardiac phase, while the minimum PVs size was in the $5 \%$ or $95 \%$ phases. However, Choi et al.[9] argued that the maximum size of the RIPV was in $35 \%$, while the minimum size of the RIPV was in $85 \%$ phase; thus, they measured the RSPV, LSPV, and LIPV in these two phases. The subject of Choi et al's study is AF and non-AF populations, while our study subject is only normal population. At the same time, Hauser et al.[17] find the timing of the PVs maximum size varied but generally occurred in ventricular diastole, and the timing of minimum PVs size also varied but generally occurred in ventricular systole, but they didn't provid specific cardiac timing points. Therefore, we believe that AF can affect the cardiac phase which the maximum and minimum of PVs is in. In normal population, Manghat NE et al.[10] directly measured the PVs size by MR cine in the end-systolic and end-diastolic stages of the ventricle, and found that the maximum size of the four PVs were all located in the ventricular end-systolic. Therefore, we can think that the ventricular end-systolic is equivalent to $45 \%$ phase of cardiac cycle in the CT reconstruction. According to Deng Wen et al's[18] physiological phases of the cardiac cycle, $40 \%$ phase corresponds to the ventricular isovolumic relaxation phase, while $50-90 \%$ corresponds to the filling phase, during which the atrial volume is larger in the early stage but later decreases. Some studies have demonstrated that during cardiac cycle, changes in the PVs ostia size are consistent with the changes in the atrial volume, and the atrial volume expansion could 
lead to an increase in the PVs ostia.[8,19] In addition, the minimum size of the four PVs ostia was observed in $5 \%$ or $95 \%$, which is equivalent to the systolic period of the cardiac phases (the atrioventricular valve is closing); at this point, the blood flow in the atrium enters the ventricle totally, and the LA volume is the smallest. Thus, the change of the PVs ostia size corroborates the behavior of the atrial volume, and any disease that causes abnormalities in the LA, such as mitral valve disease, would change the PVs' size.

Dong et al.[20] measured the maximum diameter of the four PVs in AF patients. Compared with our study, RSPV $(19.91 \pm 3.26 \mathrm{~mm})$ VS $(17.79 \pm 4.30 \mathrm{~mm})$, RIPV $(17.04 \pm 2.46 \mathrm{~mm})$ VS $15.92 \pm 3.19 \mathrm{~mm})$, LSPV $(18.93 \pm 2.78 \mathrm{~mm})$ VS $(18.54 \pm 3.44 \mathrm{~mm})$, and LIPV $(16.52 \pm 2.55 \mathrm{~mm})$ VS $(15.56 \pm 3.25 \mathrm{~mm})$, we can find that the diameter of the four PVs in normal person is higher than that in AF patients. Hence, it is obvious that AF could lead to a reduction of the PVs' size; however, Dong et al.[20] did not provide the phase of the maximum value of the PVs ostia.

\section{The correlation between traditional risk factors and PVs}

Regarding the correlation between gender and PV size, we found that the RSPV, RIPV, and LSPV differed between males and females. At the initial phase of the cardiac cycle, the average level of the three PVs size between individuals of different genders was different, and the size of these three PVs was larger in males than that in females. At different phases of the cardiac cycle, changes in the three PVs (RSPV, RIPV, and LSPV) were different, and the rate of change was also different. Notably, blood pressure and smoking affected the RSPV size. The diameter of the RSPV in hypertensive patients and smoker was small. We observed no effect of age, blood fat, blood sugar, BMI, and drinking on the PVs' size.

\section{Spatial angle of PVs}

The changing trend of the RSPV, RIPV, and LSPV cross-sectional and coronal-section angles was a straight line. For the LIPV, however, the maximum and minimum values of cross-sectional angles were observed in $45 \%$ and $5 \%$ of cardiac phases, while the maximum and minimum values on coronal-section angles were observed in $55 \%$ and $85 \%$, respectively.

Wang et al.[12] measured the angle of the PVs at a certain phase and reported that the angle is a real and stable imaging anatomical feature, which has nothing to do with gender or image features (magnetic resonance or CT).[12] In our study, we measured the spatial angles (cross-sectional and coronal-section angles) of the four PVs in 10 cardiac phases and revealed that in the initial phase of the cardiac cycle, the average value of the LIPV cross-sectional angles among individuals with different ages was different; with increasing age, the angle also increases. In different cardiac phases, individuals with different ages revealed different changes in the LIPV cross-sectional angles, and individuals with different ages had a different speed of LIPV cross-sectional angle changes. However, the cross-sectional and coronal-section angles of the RSPV, RIPV, and LSPV, as well as coronal-section angle of the LIPV, did not change considerably with the cardiac cycle change. Moreover, we found no effect of gender, blood lipid, blood glucose, BMI, drinking, and smoking on the spatial angle of these PVs. Thus, if intervening on the RSPV, 
RIPV, or LSPV, we concur with Wang et al.[12]; it is recommended to adjust the angle of the central X-ray based on the average value of the central $X$-ray angle. If intervening on the LIPV, we recommend that the pre- and postoperative image assessment should be performed at the same cardiac phase, and during the operation, the surgeon(s) must closely observe the changes in LIPV angles.

\section{Conclusion}

In conclusion, this study demonstrates that the PVs ostia size of normal person varies during cardiac phases, which is consistent with that in atria. Compared with nomal person, AF could affect the cardiac phase in which the maximum and minimum of PVs is, and it may lead to a reduction of the PVs' size alightly. This study also find that the ventricular end-systolic is equivalent to $45 \%$ phase of cardiac cycle in the CT reconstruction. In addition, the degree of PV changes in the cardiac phases varies with gender. Thus, this study suggests that the measurement of the PVs should always be at the same cardiac phase to avoid misinterpretation of variations in the cycle as stenosis. Furthermore, the angles of the RSPV, RIPV, and LSPV, is a real and stable imaging anatomical feature.

This study has some limitations worth acknowledging. First, in this study, the radiation dose was generated using CT, but the radiation dose was generally lower than the international or domestic standard. Second, this study only measured the PVs size in the normal population, and did not measure the PVs size of AF patients in 10 cardiac phases. In future study, we will continue to research the characteristics of patients' PVs with heart disease or AF.

\section{Abbreviations}

PVs, pulmonary veins; AF, atrial fibrillation; RSPV, right superior pulmonary vein; RIPV, right inferior pulmonary vein; LSPV, left inferior pulmonary vein; LIPV, left superior pulmonary vein; LA, left atrium; BMI, body mass index.

\section{Declarations}

\section{Funding}

The authors did not receive support from any organization for the submitted work.

\section{Conflicts of interest/Competing interests}

All authors certify that they have no affiliations with or involvement in any organization or entity with any financial interest or non-financial interest in the subject matter or materials discussed in this manuscript.

\section{Availability of data and material}

All the data involved in this study are available. 


\section{Code availability}

The model establishment is completed with MLwin2.36 software (version 2.36; University of Bristol, Bristol, The United Kingdom).

\section{Authors' contributions}

Jing Chen provided the ideas of this study, analyzed the data, and revised the article finall; Jing Dong collected and searched patient images, measured data, analyzed the image and data, and write articles; Hui Li statistics and analysis all the data; Yu-jiao Deng and Jin-rong Zhou analyze images.

\section{Ethics approval}

This study have been approved by our hospital ethics committee and have been performed in accordance with the ethical standards laid down in the 1964 Declaration of Helsinki and its later amendments (KY201907). Because of the retrospective nature of the study, we were not required to get signed subjects informed consent.

\section{Consent to participate}

Because of the retrospective nature of the study, we were not required to get signed subjects informed consent.

\section{Consent for publication}

All authors involved in this study agree to the publication of this article

\section{References}

1. Douglas $\mathrm{YL}$, Jongbloed MR, Deruiter MC, et al. Normal and abnormal development of pulmonary veins: state of the art and correlation with clinical entities. Int J Cardiol. 2011;147(1):13-24. https://doi.org/10.1016/j.ijcard.2010.07.004.

2. LaBourene JI, Coles JG, Johnson DJ, et al. Alterations in elastin and collagen related to the mechanism of progressive pulmonary venous obstruction in a piglet model. A hemodynamic, ultrastructural, and biochemical study. Circ Res. 1990;66(2):438-56. https://doi.org/10.1161/01.res.66.2.438.

3. Silove ED, Tavernor WD, Berry CL. Reactive pulmonary arterial hypertension after pulmonary venous constriction in the calf. Cardiovasc Res. 1972;6(1):36-44. https://doi.org/10.1093/cvr/6.1.36.

4. Hopkins RA, Hammon JW Jr, McHale PA, et al. An analysis of the pulsatile hemodynamic responses of the pulmonary circulation to acute and chronic pulmonary venous hypertension in the awake dog. Circ Res. 1980;47(6):902-10. https://doi.org/10.1161/01.res.47.6.902. 
5. Haïssaguerre M, Jaïs $P$, Shah DC, et al. Spontaneous initiation of atrial fibrillation by ectopic beats originating in the pulmonary veins. N Engl J Med. 1998;339(10):659-66. https://doi.org/10.1056/NEJM199809033391003.

6. Lickfett L, Dickfeld T, Kato R, et al. Changes of pulmonary vein orifice size and location throughout the cardiac cycle: dynamic analysis using magnetic resonance cine imaging. J Cardiovasc Electrophysiol. 2005;16(6):582-8. https://doi.org/10.1046/j.1540-8167.2005.40724.x.

7. van der Voort PH, van den Bosch H, Post JC, et al. Determination of the spatial orientation and shape of pulmonary vein ostia by contrast-enhanced magnetic resonance angiography. Europace. 2006;8(1):1-6. https://doi.org/10.1093/europace/euj006.

8. Shi Y, Mi S, Shi Y, et al. Evaluation of pulmonary vein anatomy using 256-slice computed tomography. Turk J Med Sci. 2017;47(5):1526-34. https://doi.org/10.3906/sag-1506-84.

9. Choi SI, Seo JB, Choi SH, et al. Variation of the size of pulmonary venous ostia during the cardiac cycle: optimal reconstruction window at ECG-gated multi-detector row CT. Eur Radiol. 2005;15(7):1441-5. https://doi.org/10.3906/sag-1506-84.

10. Manghat NE, Mathias HC, Kakani N, et al. Pulmonary venous evaluation using electrocardiogramgated 64-detector row cardiac CT. Br J Radiol. 2012;85(1015):965-71. https://doi.org/10.1259/bjr/79302681.

11. Chu ZG, Gao HL, Yang ZG, et al. Pulmonary veins of the patients with atrial fibrillation: dual-source computed tomography evaluation prior to radiofrequency catheter ablation. Int $\mathrm{J}$ Cardiol. 2011;148(2):245-8. https://doi.org/10.1016/j.ijcard.2010.02.076.

12. Wang J, Zhang Z, Yu W, et al. A study of images of Projective Angles of pulmonary veins. Eur J Radiol. 2009;71(3):474-9. https://doi.org/10.1016/j.ejrad.2008.05.012.

13. Merchant FM, Levy MR, Iravanian S, et al. Pulmonary vein anatomy assessed by cardiac magnetic resonance imaging in patients undergoing initial atrial fibrillation ablation: implications for novel ablation technologies. J Interv Card Electrophysiol. 2016;46(2):89-96. https://doi.org/10.1007/s10840-016-0106-9.

14. Ippolito D, Fior D, Franzesi CT, et al. Diagnostic accuracy of 256-row multidetector CT coronary angiography with prospective ECG-gating combined with fourth-generation iterative reconstruction algorithm in the assessment of coronary artery bypass: evaluation of dose reduction and image quality. Radiol Med. 2017;122(12):893-901. https://doi.org/10.1007/s11547-017-0800-4.

15. Chen J, Yang ZG, Xu HY, et al. Assessments of pulmonary vein and left atrial anatomical variants in atrial fibrillation patients for catheter ablation with cardiac CT. Eur Radiol. 2017;27(2):660-70. https://doi.org/10.1007/s00330-016-4411-6.

16. Kaseno K, Tada H, Koyama K, et al. Prevalence and characterization of pulmonary vein variants in patients with atrial fibrillation determined using 3-dimensional computed tomography. Am J Cardiol. 2008;101(11):1638-42. https://doi.org/10.1016/j.amjcard.2008.01.053.

17. Hauser TH, Yeon SB, Kissinger KV, et al (2006) Variation in pulmonary vein size during the cardiac cycle: implications for nonelectro cardiogram-gated imaging. Am Heart J 152(5):974.e1-974.e9746. 
https://doi.org/10.1016/j.ahj.2006.05.018.

18. Deng W, Yang ZG, Peng LQ, et al. Morphological and dynamic features of normal mitral valve evaluated by dual-source computed tomography. Int J Cardiol. 2010;145(3):633-6. https://doi.org/10.1016/j.ijcard.2010.09.077.

19. Bowman AW, Kovács SJ. Prediction and assessment of the time-varying effective pulmonary vein area via cardiac MRI and Doppler echocardiography. Am J Physiol Heart Circ Physiol. 2005;288(1):H280-6. https://doi.org/10.1152/ajpheart.00713.2004.

20. Dong QQ, Yang WY, Sun YP, et al. Comparison of transthoracic echocardiography with computed tomography in evaluation of pulmonary veins. BMC Cardiovasc Disord. 2019;19(1):315. https://doi.org/10.1186/s12872-019-01272-8.

\section{Tables}

Table 1. Subject characteristic

\begin{tabular}{|ll|}
\hline & $\mathrm{n}=101$ \\
\hline Male/Female & $51 / 50$ \\
\hline Age, mean \pm SD, years & $50.79 \pm 10.10$ \\
\hline BMI & $23.61 \pm 3.28$ \\
\hline hypertension & 24 \\
\hline hyperlipemia & 35 \\
\hline hypercemia & 18 \\
\hline somking & 25 \\
\hline drinking & 10 \\
\hline
\end{tabular}

$\mathrm{BMI}$, body mass index; data are presented as mean \pm standard deviation or $\mathrm{n}$.

Table 2. The comparisons of RSPV and RIPV's size between cardiac phases 


\begin{tabular}{|c|c|c|c|c|}
\hline & diameter & area & cross-sectional angle & coronal-sectional angle \\
\hline \multicolumn{5}{|c|}{ RSPV } \\
\hline $5 \%$ & $18.02 \pm 2.93$ & $201.73 \pm 66.20$ & $18.30 \pm 8.29$ & $25.10 \pm 8.73$ \\
\hline $15 \%$ & $18.57 \pm 3.06$ & $211.80 \pm 65.00$ & $18.08 \pm 8.14$ & $24.32 \pm 8.16$ \\
\hline $25 \%$ & $19.10 \pm 3.00$ & $239.61 \pm 73.31$ & $17.04 \pm 7.96$ & $23.73 \pm 8.14$ \\
\hline $35 \%$ & $19.59 \pm 3.35$ & $268.66 \pm 82.35$ & $16.74 \pm 7.71$ & $23.39 \pm 8.37$ \\
\hline $45 \%$ & $19.91 \pm 3.26$ & $275.38 \pm 83.29$ & $16.41 \pm 7.55$ & $23.72 \pm 8.22$ \\
\hline $55 \%$ & $19.61 \pm 3.10$ & $255.14 \pm 77.59$ & $17.08 \pm 7.75$ & $23.96 \pm 8.28$ \\
\hline $65 \%$ & $19.60 \pm 3.13$ & $240.42 \pm 68.58$ & $16.97 \pm 7.74$ & $23.94 \pm 8.35$ \\
\hline $75 \%$ & $19.73 \pm 3.12$ & $244.71 \pm 69.45$ & $16.81 \pm 7.86$ & $23.78 \pm 8.48$ \\
\hline $85 \%$ & $19.42 \pm 3.10$ & $234.29 \pm 68.19$ & $17.20 \pm 7.81$ & $24.28 \pm 8.61$ \\
\hline $95 \%$ & $17.28 \pm 2.82$ & $196.33 \pm 64.18$ & $17.79 \pm 24.9$ & $24.91 \pm 8.68$ \\
\hline \multicolumn{5}{|l|}{ RIPV } \\
\hline $5 \%$ & $15.58 \pm 2.25$ & $175.12 \pm 50.17$ & $29.30 \pm 11.69$ & $18.06 \pm 9.93$ \\
\hline $15 \%$ & $15.93 \pm 2.33$ & $183.01 \pm 51.47$ & $29.46 \pm 11.42$ & $17.63 \pm 9.42$ \\
\hline $25 \%$ & $16.52 \pm 2.35$ & $194.68 \pm 55.26$ & $30.04 \pm 11.08$ & $17.88 \pm 9.40$ \\
\hline $35 \%$ & $16.86 \pm 2.48$ & $206.67 \pm 59.32$ & $30.08 \pm 11.20$ & $17.53 \pm 9.45$ \\
\hline $45 \%$ & $17.04 \pm 2.46$ & $210.74 \pm 58.80$ & $30.28 \pm 11.19$ & $17.62 \pm 9.45$ \\
\hline $55 \%$ & $16.79 \pm 2.43$ & $201.53 \pm 56.20$ & $29.77 \pm 11.14$ & $17.82 \pm 9.39$ \\
\hline $65 \%$ & $16.54 \pm 2.35$ & $197.59 \pm 53.64$ & $29.54 \pm 10.93$ & $17.34 \pm 9.38$ \\
\hline $75 \%$ & $16.58 \pm 2.45$ & $198.80 \pm 56.64$ & $29.91 \pm 11.20$ & $17.72 \pm 9.52$ \\
\hline $85 \%$ & $16.22 \pm 2.55$ & $194.04 \pm 60.51$ & $29.85 \pm 11.12$ & $17.66 \pm 9.55$ \\
\hline $95 \%$ & $15.30 \pm 2.48$ & $169.92 \pm 54.11$ & $29.56 \pm 11.22$ & $17.77 \pm 9.93$ \\
\hline
\end{tabular}

Data are presented as mean \pm standard deviation; RSPV, the right inferior pulmonary vein; RIPV, the right inferior pulmonary vein.

Table 3. The comparisons of LSPV and LIPV's size between cardiac phases 


\begin{tabular}{|c|c|c|c|c|}
\hline & diameter & area & cross-sectional angle & coronal-sectional angle \\
\hline \multicolumn{5}{|c|}{ LSPV } \\
\hline $5 \%$ & $16.79 \pm 2.79$ & $169.21 \pm 48.55$ & $16.02 \pm 8.60$ & $34.23 \pm 11.96$ \\
\hline $15 \%$ & $17.01 \pm 2.65$ & $175.68 \pm 51.43$ & $16.46 \pm 8.04$ & $33.71 \pm 12.12$ \\
\hline $25 \%$ & $17.80 \pm 2.73$ & $199.10 \pm 56.80$ & $16.95 \pm 8.12$ & $33.09 \pm 12.31$ \\
\hline $35 \%$ & $18.66 \pm 2.79$ & $216.72 \pm 60.23$ & $17.16 \pm 8.38$ & $32.80 \pm 12.74$ \\
\hline $45 \%$ & $18.93 \pm 2.78$ & $224.22 \pm 62.53$ & $17.17 \pm 8.31$ & $33.09 \pm 12.30$ \\
\hline $55 \%$ & $18.46 \pm 2.63$ & $204.98 \pm 54.99$ & $16.65 \pm 8.29$ & $32.29 \pm 12.02$ \\
\hline $65 \%$ & $18.34 \pm 2.85$ & $191.91 \pm 50.66$ & $15.87 \pm 8.10$ & $31.13 \pm 11.80$ \\
\hline $75 \%$ & $18.47 \pm 2.87$ & $192.10 \pm 52.35$ & $15.77 \pm 8.20$ & $31.10 \pm 11.68$ \\
\hline $85 \%$ & $18.23 \pm 2.86$ & $191.69 \pm 52.96$ & $15.75 \pm 8.21$ & $31.47 \pm 12.17$ \\
\hline $95 \%$ & $16.68 \pm 2.62$ & $171.99 \pm 47.24$ & $16.15 \pm 8.11$ & $32.70 \pm 12.27$ \\
\hline \multicolumn{5}{|l|}{ LIPV } \\
\hline $5 \%$ & $15.11 \pm 2.23$ & $126.93 \pm 41.23$ & $20.18 \pm 8.76$ & $20.24 \pm 10.14$ \\
\hline $15 \%$ & $15.53 \pm 2.43$ & $130.40 \pm 43.00$ & $20.49 \pm 9.11$ & $19.98 \pm 9.73$ \\
\hline $25 \%$ & $16.00 \pm 2.58$ & $144.32 \pm 53.97$ & $21.23 \pm 9.04$ & $20.38 \pm 9.63$ \\
\hline $35 \%$ & $16.44 \pm 2.77$ & $152.02 \pm 59.17$ & $21.80 \pm 8.87$ & $20.47 \pm 9.66$ \\
\hline $45 \%$ & $16.52 \pm 2.55$ & $154.84 \pm 60.52$ & $22.18 \pm 9.06$ & $20.44 \pm 9.49$ \\
\hline $55 \%$ & $16.39 \pm 2.48$ & $146.87 \pm 51.56$ & $21.87 \pm 8.80$ & $20.59 \pm 10.01$ \\
\hline $65 \%$ & $16.27 \pm 2.51$ & $142.93 \pm 48.04$ & $21.48 \pm 8.31$ & $20.11 \pm 9.37$ \\
\hline $75 \%$ & $16.30 \pm 2.35$ & $144.16 \pm 49.22$ & $21.25 \pm 8.31$ & $19.91 \pm 9.46$ \\
\hline $85 \%$ & $16.12 \pm 2.47$ & $141.82 \pm 48.44$ & $20.72 \pm 7.94$ & $19.38 \pm 9.02$ \\
\hline $95 \%$ & $15.26 \pm 2.10$ & $130.27 \pm 42.20$ & $20.20 \pm 8.18$ & $19.65 \pm 9.33$ \\
\hline
\end{tabular}

Data are presented as mean \pm standard deviation; LSPV, the left inferior pulmonary vein; LIPV, the left inferior pulmonary vein.

Figures 


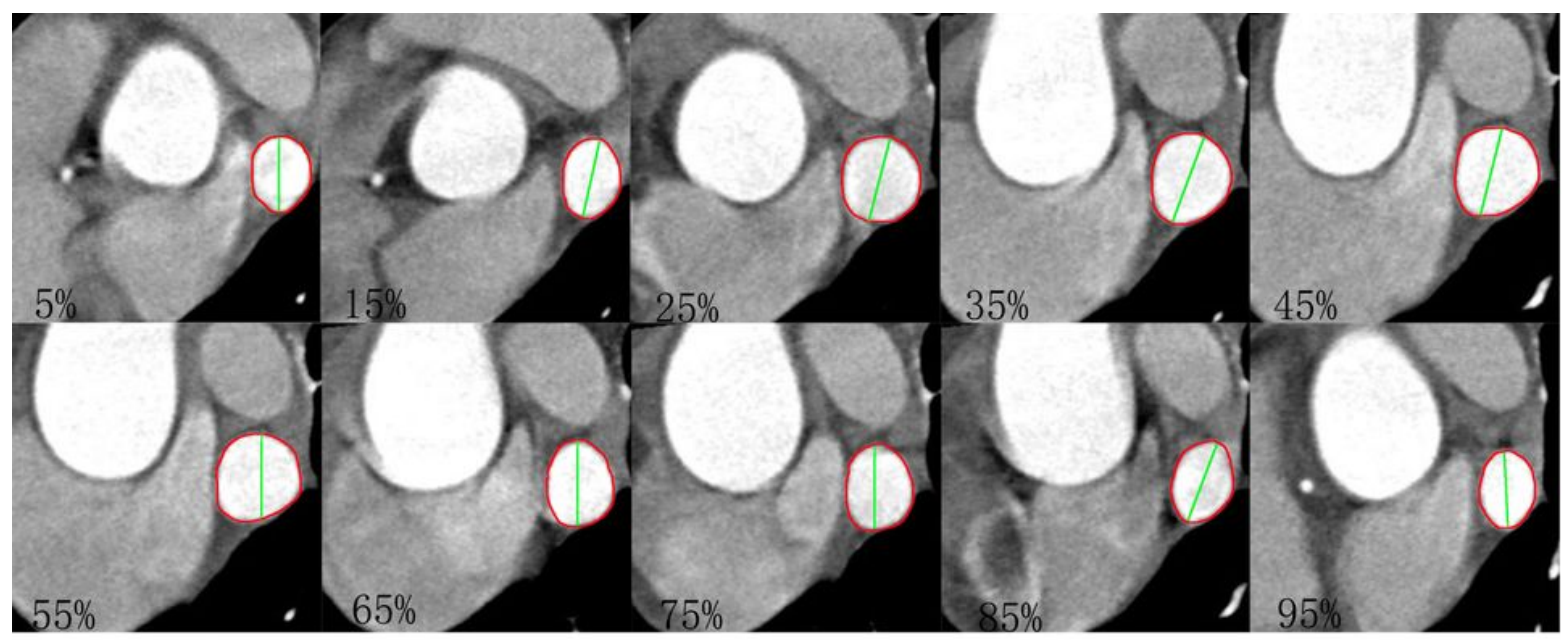

\section{size}

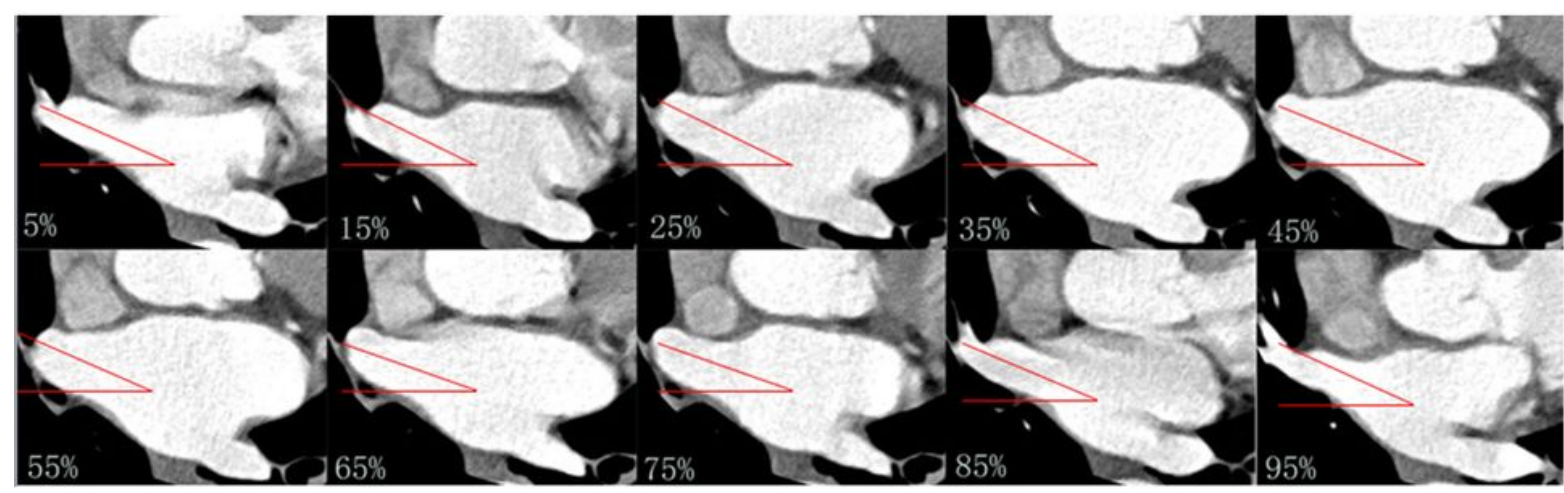

cross-section

angel
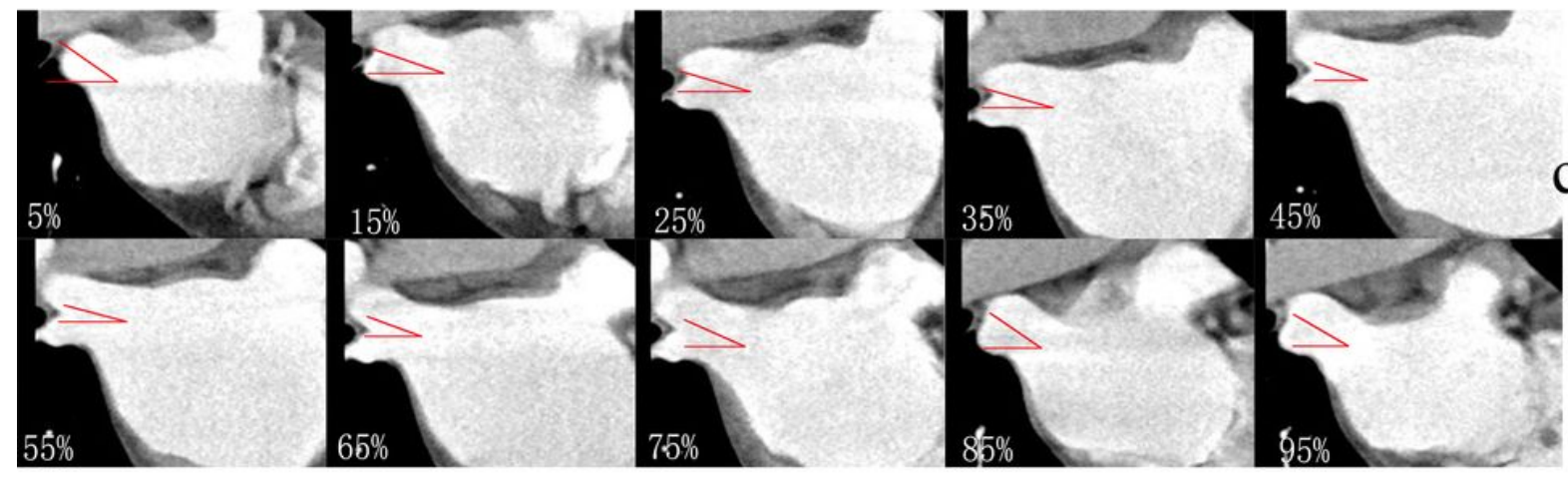

coronal-section angel

\section{Figure 1}

The original images were reconstructed from $5 \%$ to $95 \%$ cardiac phases at $10 \%$ intervals. The reconstructed images were transferred to the post-processing software (IntelliSpace Portal Workspace; Philips) and loaded into the cardiac viewer application, which enabled three perpendicular axes to be adjusted simultaneously to attain perpendicular imaging planes for measurement. Two experienced radiologists (with 2- and 5-year experience in the interpretation of cardiac CT images), blinded to clinical data, independently reviewed the CT images on a dedicated post-processing workstation. They measured the diameter, area, cross-sectional angle, and coronal-section angle of the four PVs ostia. As shown in Fig. 1, we measured the diameter and area of the sinus ostium at the PVs ostia, followed by measuring the cross-sectional and coronal-section angles of the PVs ostia, respectively. 

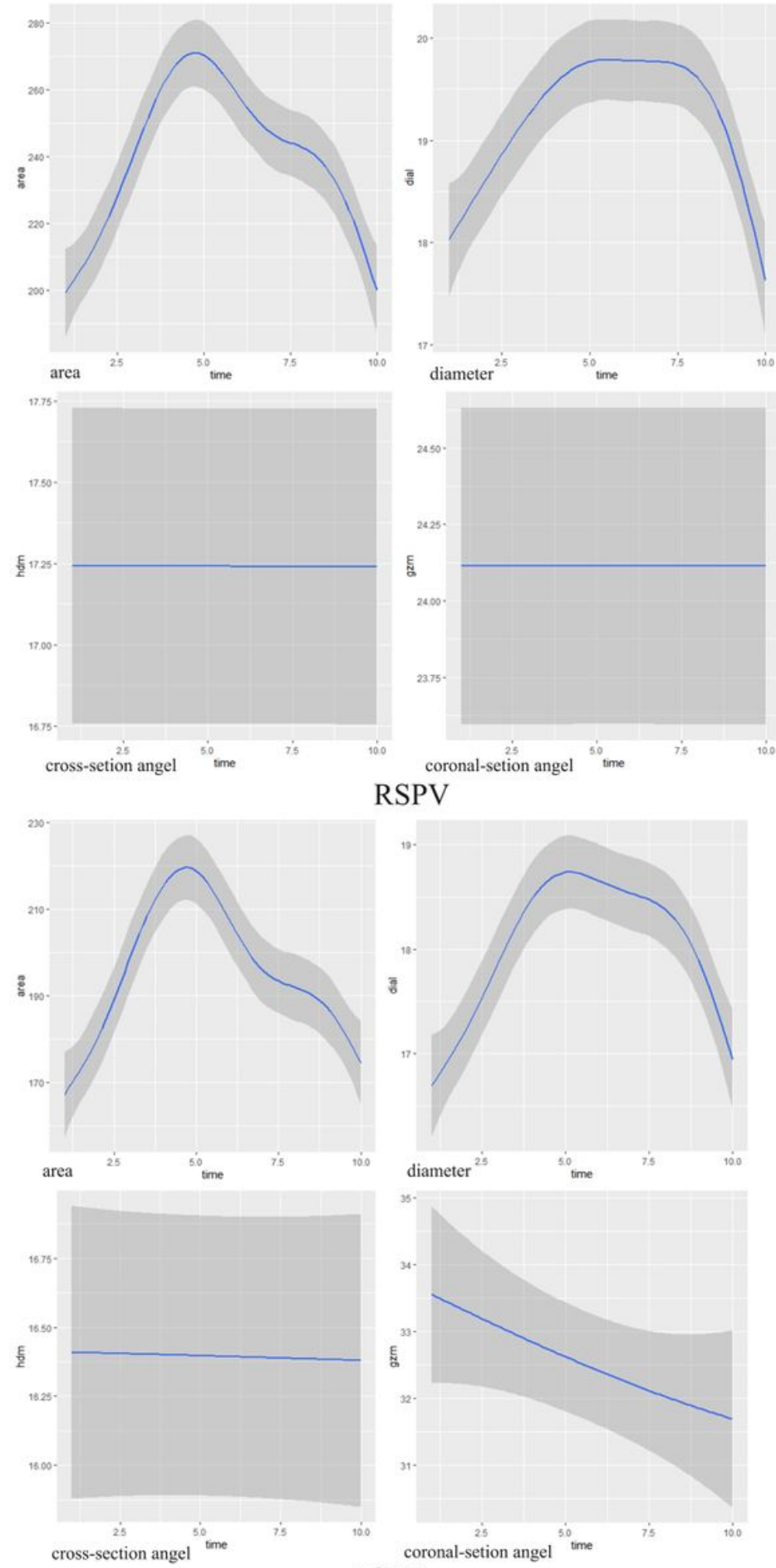

LSPV
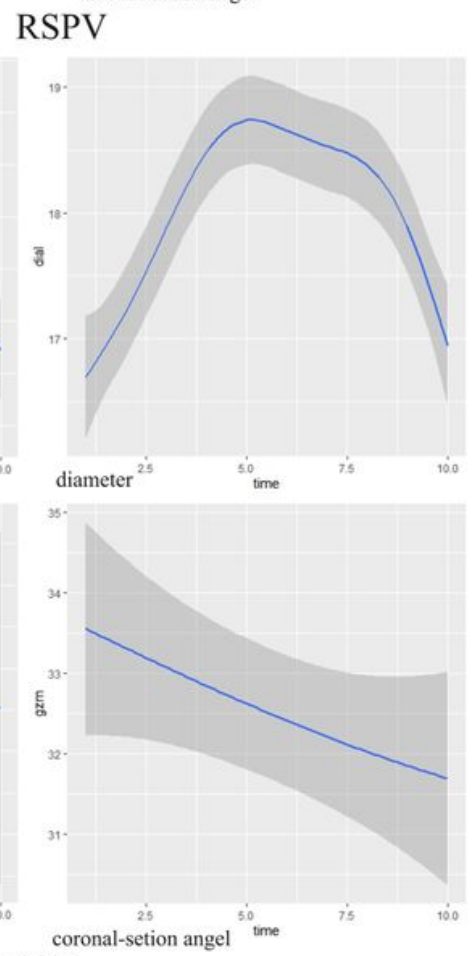
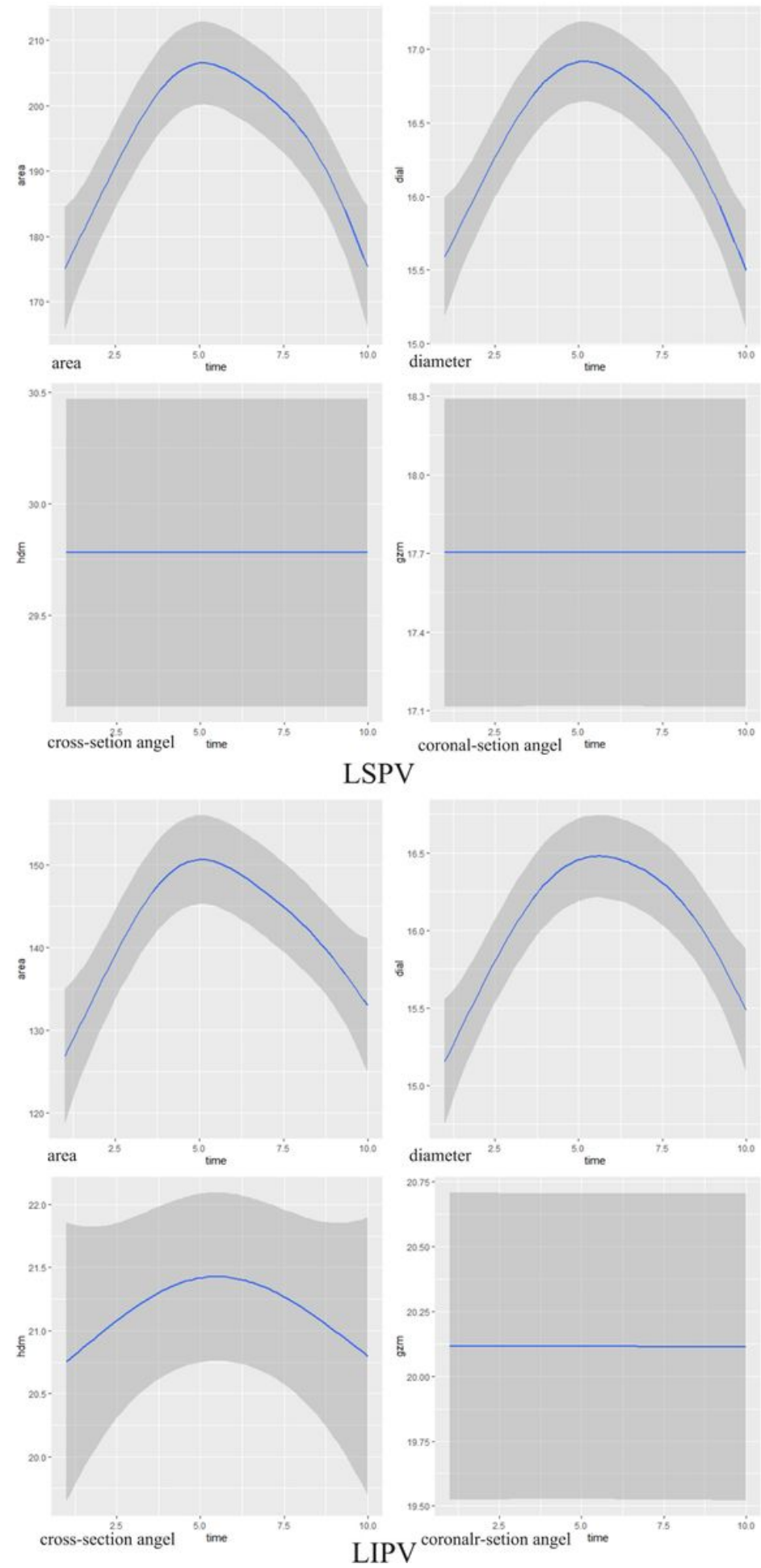

\section{Figure 2}

Figure 2 shows the difference in the cross-sectional angle of the LIPV. We used a quadratic model to fit the trend of the LIPV cross-sectional angle with cardiac phases.

\section{Supplementary Files}

This is a list of supplementary files associated with this preprint. Click to download. 
- SI.docx

Page 16/16 